\title{
Interpolation Based Control for reference tracking under constraints
}

\author{
Martin Soyer ${ }^{1,2}$, Sorin Olaru ${ }^{2}$, Zhou Fang ${ }^{1}$
}

\begin{abstract}
This article introduces an adaptation of the Interpolation Based Control technique developed recently for the constraint regulation. The aim of this work is to address the particularities of the trajectory tracking control problems in the presence of constraints. In this framework, the main challenge comes from translation of the constraint set as a result of the evolution of the trajectory to be tracked. Even if the trajectory satisfies the dynamical constraints, and those dynamics are linear, the translation of the constraints set leads to topological changes in the controllable regions. The associated controlledinvariant sets are difficult to compute online and alternative techniques which avoid set-iterations at each sampling instant need to be devised. The main idea in the present contribution is to build on the IBC regulation philosophy which proved the computational attractiveness and to adapt its ingredients to the trajectory tracking by constructing a virtual feasible trajectory with particular properties and enable the interpolation on scalable version of the controlled invariant sets designed initially for regulation. As a secondary objective, the analysis the shape of the real trajectory and the computational burden points to the possible parametrization of the virtual trajectory and enhance the proposed methodology.
\end{abstract}

\section{INTRODUCTION}

Constrained trajectory tracking is a topic which received the interest over more than 30 years [1], [2], [3], often in connection with Model Predictive Control [4], [5], [6]. The underlying problem in the constraint trajectory tracking is the description of the feasible range of trajectories given a predefined stabilizing control able to handle the constraints and guarantee the constraint stabilization [7], [8]. Once this objective is achieved, the selection among the feasible trajectories of a suitable candidate is usually made with respect to an optimization-based and carries the name of reference-governor, reference-management or virtual trajectory selection.

In the field of constrained control, the so-called Interpolation Based Control (IBC) technique received [9] the interest as a fast optimization-based design technique to avoid the classical Model Predictive Control formulations all by preserving the essential features: recursive feasibility, stability, continuity and manageable performance certificates for the unconstrained control regions. Originaly, IBC has been established [10] to enhance Vertex Control [11] which was capable to stabilize a constrained system stating from an initial controlled invariant set which can approximate the

\footnotetext{
1,2 Renault Technocentre, AD Simulation \& VR center, Guyancourt,CentraleSuplec, L2S, Gif-sur-Yvette, France martin. soyererenault. com

1 Renault Technocentre, AD Simulation \& VR center, Guyancourt, France zhou. fangerenault. com

2 CentraleSuplec, L2S, Gif-sur-Yvette, France sorin.olaru@centralesupelec.fr
}

maximal controlled invariant set. The vertex control used the full control authority only on the boundary of the controlled invariant set and thus the performance when approaching the equilibrium was rather poor. IBC addressed this issue by composing in a smooth manner [12] the vertex controller with a high gain control in order to find a control law that respects the system constraints and avoids the high computational complexity of optimization-based methods for constrained control such as Model Predictive Control.

Coming back to the structural properties of the Vertex Control, its initial formulations were building on discrete time invariant linear system ( latter extended [13] to time varying systems). For a linear system with polyhedral constraints, one can define a polyhedral controlled invariant set for which it exists, for each vertex, an admissible control action that pushes the state away from the frontier and leads the state to the origin in finite time. Thus, as each state of the polyhedron can be written as a convex combination of vertex, a control law can be found for every state of the invariant set by means of a simple LP problem. In IBC [14] a second controlled invariant set, associated to a high gain (high performance in the unconstrained case) is considered in the design. The convex decomposition between a high gain unconstrained linear feedback law in the neighborhood of the origin and a low gain Vertex law near the frontiers of the invariant set was shown to preserve the LP formulation and add the performance on top of the large region of attraction. All these developments reach a maturity for the constrained regulation around the origin and has been applied in different domains [15], [16] with different groups developing the associated design tools [17]. Interesting though, the trajectory tracking was not addressed in these works and represents an important aspect to complete the technique with the capabilities of a generic constrained control routine.

In this paper an IBC trajectory tracking problem is formulated and solved starting from the classical ingredients of a IBC regulation. The design principle is inherited from reference governor mechanisms which design an admissible reference with respect to the static constraints and for which the regulation capabilities of the IBC can be fully exploited. For the class of linear time-invariant systems, the generation of an admissible virtual trajectory is done in conjunction with a scaling mechanism for the controlled invariant sets involved in the IBC design. Globally the tracking IBC solution is shown to preserve the LP structure and thus presents attractive features for real-time implementation. The proposed technique is formally presented together with the feasibility proof and a series of enhancements are presented along with numerical examples. 


\section{A. Notations}

A Polytope $P$ in the H-Representation is a set $P=$ $\left\{x \in \mathbb{R}^{n} / F x \leq g\right\}$ where $F \in \mathbb{R}^{p \times n}$ and $g \in \mathbb{R}^{p}$. A Polyhedron denotes a bounded polytope. A Polyhedron $P$ in $V$-Representation is a set $P=\left\{\sum_{i}^{n} \lambda_{i} v_{i} / \forall i \in \llbracket 0, n \rrbracket \lambda_{i} \geq\right.$ $0\}$ where $\left(v_{1}, \ldots, v_{n}\right)$ are vertex of the polyhedron. The Minkowski sum of two sets $A \subset \mathbb{R}^{m}$ and $B \subset \mathbb{R}^{p}$ is the set of all combinations of elements of $A$ and $B$. It is denoted as $A \oplus B=\{a+b / a \in A, b \in B\}$. The Minkowski difference is defined as $A \ominus B=\left\{x \in \mathbb{R}^{n} /\{x\} \oplus B \subset A\right\}$. The weighted 2-norm $\|\cdot\|_{Q}$ is defined as follow : $\forall x \in \mathbb{R}^{n}\|x\|_{Q}^{2}=x^{T} Q x$ where $Q \in \mathbb{R}^{n \times n}$ is positive definite.

\section{B. Definitions}

Definition I.1 (Positively Invariant Set). A set $\Omega$ is said to be positively invariant for the system $x_{k+1}=f\left(x_{k}\right)$ if $x_{0} \in$ $\Omega \Rightarrow x_{k} \in \Omega \forall k \in \mathbb{N}$.

Definition I.2 (Positively Invariant and Admissible Set). Consider a discrete linear system $x_{k+1}=f\left(x_{k}, u_{k}\right)$ subject to a linear feedback controller $u_{k}=\mathcal{K}\left(x_{k}\right)$, a set $\Omega$ is said to be Positively Invariant Set Admissible Set with respect to states and control constraints if $x_{k} \in \Omega \Rightarrow x_{k+1} \in$ $\Omega$ and $\mathcal{K}\left(x_{k}\right) \in \mathbb{U}$, where $\mathbb{U}$ represents the input constraints set.

\section{INTERPOLATION BASED CONTROL}

\section{A. Principle}

Given constrained linear discrete-time system :

$$
x(k+1)=A x(k)+B u(k)
$$

where $x \in \mathbb{X} \subset \mathbb{R}^{n}, u \in \mathbb{U} \subset \mathbb{R}^{m}, A \in \mathbb{R}^{n \times n}, B \in \mathbb{R}^{n \times m}$. $\mathbb{X}$ and $\mathbb{U}$ are sets of polyhedral constraints respectively on states and control vector : $\mathbb{X}=\left\{x \in \mathbb{R}^{n} / F_{x} x \leq g_{x}\right\}$ and $\mathbb{U}=\left\{u \in \mathbb{R}^{m} / F_{u} u \leq g_{u}\right\}$ both containing the origin in their interior. Under controllability assumptions, there exist admissible control-invariant sets. Consider two such sets $\Omega^{o} \subset \mathbb{X}$ and $\Omega^{v} \subset \mathbb{X}$ which are convex compacts and satisfy :

$$
\Omega^{o} \subset \Omega^{v} \subset \mathbb{X}
$$

$\Omega^{\circ}$ will be denoted inner set generally associated with a high gain control law and $\Omega^{v}$ the outer set which is intended to approximate the maximal controllable set within $\mathbb{X}$. So for every initial state $x_{0} \in \Omega^{v}$ there exists a control sequence that leads the system to the origin and consequently to $\Omega^{\circ}$ in a finite number of steps.

$I B C$ principle: at each step $k \in \mathbb{N}$, given the measurement of the current state, the controller finds a convex combination of states in the inner and respectively outer controlled invariant set such that :

$$
x_{k}=c_{k} x_{k}^{v}+\left(1-c_{k}\right) x_{k}^{o}
$$

Where $x_{k}^{v} \in \Omega^{v}, x_{k}^{o} \in \Omega^{o}$ and $c_{k} \in[0,1]$. Once the convex decomposition is available the IBC can be exploited as stated in the next result.
Theorem II.1. Given the system (1) and the controlled invariant sets $\Omega^{o}$ and $\Omega^{v}$ with $\Omega^{o} \subset \Omega^{v}$, the following control law :

$$
u_{k}=c_{k} u_{k}^{v}+\left(1-c_{k}\right) u_{k}^{o}
$$

where $u_{k}^{o}$ is a control action in $\Omega^{o}$ and $u_{k}^{v}$ is a control law in $\Omega^{v}$ and the convex factor $c_{k}$ is the solution of the following optimization problem :

$$
\begin{array}{cl}
\underset{\left(x_{k}^{v}, x_{k}^{o}, c_{k}\right)}{\operatorname{minimize}} & c_{k} \\
\text { subject to } & x_{k}^{v} \in \Omega^{v}, \\
& x_{k}^{o} \in \Omega^{o}, \\
& x_{k}=c_{k} x_{k}^{v}+\left(1-c_{k}\right) x_{k}^{o}, \\
& c \in[0,1] .
\end{array}
$$

is admissible and the origin is a stable equilibrium for the closed-loop system with a basin of attraction $\Omega^{v}$.

Proof : The argument is based on the use of the convex factor $c_{k}$ as a Lyapunov function. We point the reader to [14] for the complete proof but we emphasize that this problem is recursively feasible for all $x_{0} \in \Omega^{v}$ in the virtue of the control invariance properties of this set.

A geometric interpretation is proposed on Figure 1 and the bloc diagram of the Figure 2 summarizes the IBC principle.

Theorem II.2. The optimal solution $\left(x_{k}^{v}, x_{k}^{o}, c_{k}\right)$ of the optimization problem (5) is such that $x_{k}^{v}$ and $x_{k}^{o}$ are respectively on the frontier of $\Omega^{v}$ and $\Omega^{o}$.

Proof: The proof is given in [10].

Remark: For the sake of simplicity we present here an IBC with 2 sets $\left(\Omega^{v}, \Omega^{o}\right)$ but note that the technique can be generalized to multiple sets : $\left(\Omega^{v_{1}}, \Omega^{v_{2}}, \ldots, \Omega^{v_{p}}, \Omega^{o}\right)$.

\section{B. Problem statement}

The computational advantages of the IBC control with respect to alternative optimization-based constrained control as MPC have been demonstrated in theory [12], [14] and applications [17], [16]. However, the existing theory and the reported applications are dealing exclusively with the

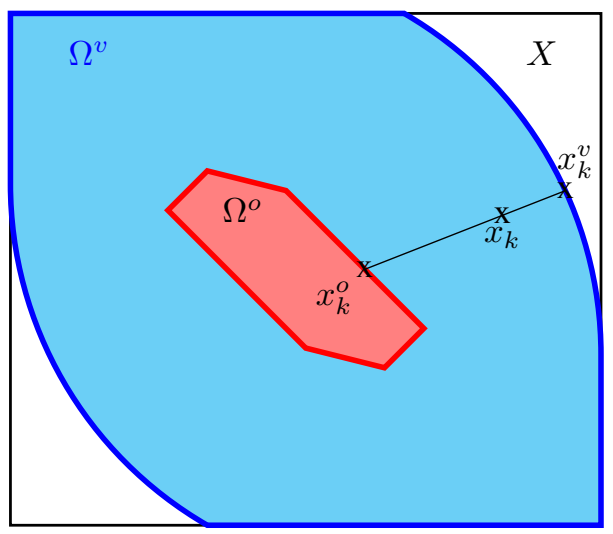

Fig. 1. IBC Principle 


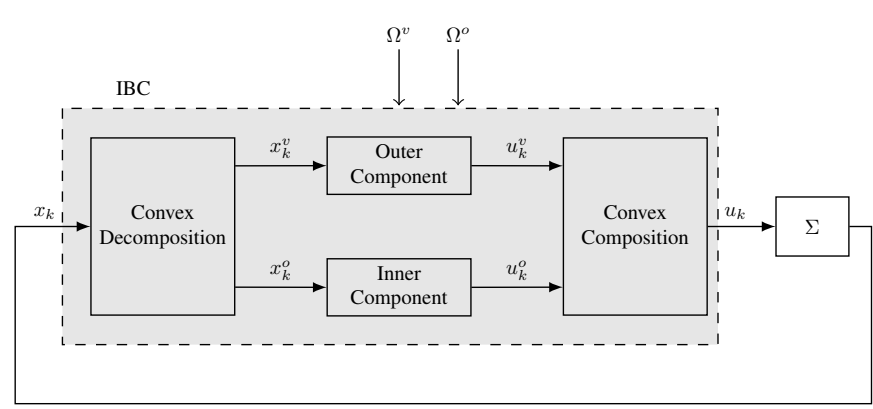

Fig. 2. Bloc diagram of IBC Principle

stabilization of the origin but didn't investigate the IBC properties for trajectory tracking. This paper aims to propose an adaptation of IBC principles in order to enhance the tracking of an arbitrary reference trajectory, allowing the statement of the control problem with respect to to an arbitrary reference which will be subsequently replaced by an admissible trajectory. The main idea is to propose a reference governor based algorithm that generates a virtual feasible reference adapted to IBC regulation. Given a reference trajectory $\left(x_{k}^{r e f}, \ldots x_{k+N}^{r e f}\right)$, the controller has to find $u_{k} \in \mathbb{U}$ such that $x$ tracks $x^{r e f}$ by satisfying the state constraints. The usual solution is an optimization-based controller minimizing the tracking error on a finite horizon :

$$
\begin{array}{cl}
\underset{\left(u_{k}, \ldots, u_{k+N-1}\right)}{\operatorname{minimize}} & \sum_{i=1}^{N}\left\|x_{k+i}^{r e f}-x_{k+i}\right\|_{Q}^{2}+\left\|u_{k+i}\right\|_{R}^{2} \\
\text { subject to } & x_{k+i} \in \mathbb{X} \forall i \in \llbracket 1, N \rrbracket
\end{array}
$$

In the case the reference trajectory is not admissible, the literature tends to propose MPC-based procedure with reference governor to compute an admissible trajectory to track. This paper proposes an IBC-Reference governor coupling with a restrained prediction of 2 steps to minimize the computational cost and which is feasible. The reference trajectory is not necessarly known over a prediction horizon in real-time systems, IBC for tracking may handle a relevent quantity of information with respect to real-time systems.

\section{IBC FOR TRACKING}

To introduce the IBC-based tracking procedure, we recall a theorem on homogeneity of controllable invariant sets that will be used further. The main results are then presented first by principles and subsequently through the mathmematical formulations.

\section{A. Instrumental theorems}

The challenge is the translation of the set which may lead to the loss of control invariance in the presence of input and fixed constraints. In the following procedure, the translation of the sets will be accompanied by a re-scaling of those sets.

Theorem III.1 (Homogeneity of Controlled Invariance). If a given set $\Omega$ is controlled invariant with respect to (1), $\mathbb{U}$ and $\mathbb{X}$ as input and state constraints sets, then for all $\alpha \in[0,1]$ $\alpha \Omega$ is a controlled invariant set with an admissible control action in $\alpha \mathbb{U}$.
Proof: Let $\Omega$ a controlled invariant set and $\alpha \in[0,1]$. If $\alpha=0$, then $\alpha \Omega=\{0\}$ and the trivial choice $u=0$ renders the set $\alpha \Omega$ controlled invariant. For $\alpha \in] 0,1]$ and $\xi_{0} \in \alpha \Omega$, there exists $x_{0} \in \Omega$ such that $x_{0}=\xi_{0} / \alpha$. Due to the controlled invariance of $\Omega$, there exists $u_{0} \in \mathbb{U}$ such that

$$
\begin{aligned}
x_{1} & =A x_{0}+B u_{0} \in \Omega \\
\xi_{1} / \alpha & =A\left(\xi_{0} / \alpha+B u_{0}\right) \in \Omega \\
\xi_{1} & =A \xi_{0}+B \alpha u_{0} \in \alpha \Omega
\end{aligned}
$$

By writing $v_{0}=\alpha u_{0}$ :

There exists $v_{0} \in \alpha \mathbb{U}$ such that $\xi_{1}=A \xi_{0}+B v_{0} \in \alpha \Omega$

\section{B. Principle of IBC for tracking}

The Interpolation Based Tracking (IBT) strategy is to find a control action $u_{k}$ to follow the reference and $u_{k}$ is a trade-off between a control action $\tilde{u}_{k}$ that generates a new virtual feasible reference trajectory for the system and an action $v_{k}$ that compensates the tracking error between the new reference and the current state with an IBC regulation with $u_{k}=\tilde{u}_{k}+v_{k}$. The procedure is illustrated in the fig.3.

Stage 1 : Reference Governor

- At step $\mathrm{k}$, the reference governor has to find $\tilde{u}_{k}$ and a scaling factor $\alpha_{k}$ to deduce a virtual state $\tilde{x}_{k+1 \mid k}$ in order to minimize the error between the virtual state and the real reference at the next step (5a).

- The virtual state will be imposed the same dynamics as the system (5b) at least for the current state.

- The virtual state is in the neighborhood of the current state such that the error $\varepsilon_{k}=x_{k}-\tilde{x}_{k}$ is in the re-scaled set $\alpha_{k} \Omega^{v}(5 \mathrm{c})$ which is a controlled invariant according to Theorem II.1.

- This re-scaled controlled invariant centered on the virtual state has to be in the global controlled invariant set $\Omega^{v}$ to respect constraints $(5 \mathrm{~d})$.

- The contribution of the reference governor is higher if the current state $x_{k}$ and the virtual state $\tilde{x}_{k \mid k}$ are close i.e if the scaling factor $\alpha_{k}$ is small (5e).

- This action has to lead the virtual state in another feasible neighborhood (5f).

Stage 2: IBC regulation on the error

The IBC prodecure is applied to the dynamics : $\varepsilon_{k}=$ $x_{k}-\tilde{x}_{k}$ in the re-scaled sets $\alpha \Omega^{o}$ and $\alpha \Omega^{v}$. A regulation action $v_{k}$ is found based on standard IBC.

Stage 3 : Combination

The applied control action $u_{k}$ is the combination of the reference governor control vector $\tilde{u}_{k}$ and the regulation action $v_{k}$.

A bloc diagram of the procedure is proposed on Figure 3 and a geometric interpretation of the reference governor principle is given in Figure 4.

\section{Mathematical formulation}

Stage 1: Assume the current state $x_{k}$ in the controlled invariant $\Omega^{v}$, the reference governor has to solve the following optimization problem : 


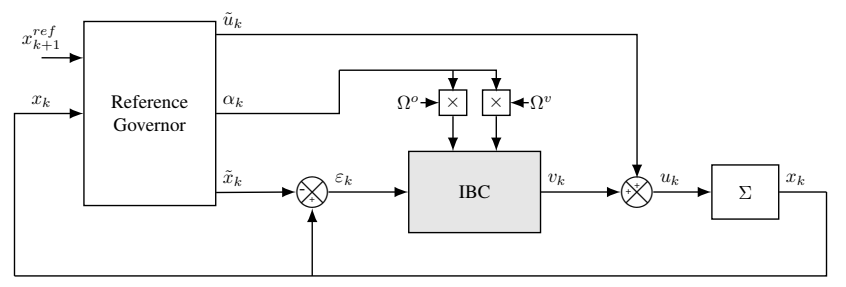

Fig. 3. Bloc diagram of IBC for tracking

$$
\left[\begin{array}{c}
\tilde{u}_{k} \\
\alpha_{k}
\end{array}\right]\left(x_{k}^{r e f}, x_{k}, \tilde{x}_{k}\right)=\underset{\left(\tilde{u}_{k}^{T}, \alpha_{k}\right)}{\arg \min }\left\|x_{k+1}^{r e f}-\tilde{x}_{k+1 \mid k}\right\|_{Q}^{2}
$$

subject to

$$
\begin{aligned}
& \tilde{x}_{k+1 \mid k}=A \tilde{x}_{k \mid k}+B \tilde{u}_{k}, \\
& x_{k} \in\left\{\tilde{x}_{k \mid k}\right\} \oplus \alpha_{k} \Omega^{v}, \\
& \left\{\tilde{x}_{k \mid k}\right\} \oplus \alpha_{k} \Omega^{v} \subset \Omega^{v}, \\
& \tilde{u}_{k} \in\left(1-\alpha_{k}\right) \mathbb{U} \\
& \left\{\tilde{x}_{k+1 \mid k}\right\} \oplus \alpha_{k} \Omega^{v} \subset \Omega^{v}
\end{aligned}
$$

The results of the optimization (7) lead to a solution $\tilde{u}_{k}$ wich provides practically a virtual trajectory $\tilde{x}_{k}$ that satisfy the dynamical constraints of the internal model (1). Thus, Stage 2 uses $\alpha_{k}$ and $\tilde{x_{k}}$ as parameters.

Stage 2 : The regulation problem is addressed through an IBC applied to the error between the current state and the intermediary state : $\varepsilon_{k}=x_{k}-\tilde{x}_{k}$. The optimization performs the convex decomposition of the error between the re-scaled outer set $\alpha_{k} \Omega^{v}$ and the re-scaled inner set $\alpha_{k} \Omega^{o}$.

$$
\begin{gathered}
{\left[\begin{array}{c}
\varepsilon_{k}^{v} \\
\varepsilon_{k}^{o} \\
c_{k}
\end{array}\right]\left(\tilde{x}_{k}, x_{k}, \alpha_{k}\right)=\underset{\left(\varepsilon_{k}^{v}, \varepsilon_{k}^{o}, c_{k}\right)}{\arg \min } c_{k}} \\
\text { subject to } \\
\varepsilon_{k}^{v} \in \alpha_{k} \Omega^{v}, \\
\varepsilon_{k}^{o} \in \alpha_{k} \Omega^{o}, \\
\\
\varepsilon_{k}=c_{k} \varepsilon_{k}^{v}+\left(1-c_{k}\right) \varepsilon_{k}^{o}, \\
\\
c_{k} \in[0,1]
\end{gathered}
$$

Optimization (8) provides regulation errors in inner and outer sets and the convex factor which defines which regulation action is preponderant, those results are used in Stage 3.

Stage 3: The control action at step $\mathrm{k}$ is computed with the following formula :

$$
u_{k}=\tilde{u}_{k}+\underbrace{c_{k} v_{k}^{v}\left(\varepsilon_{k}^{v}\right)+\left(1-c_{k}\right) v_{k}^{o}\left(\varepsilon_{k}^{o}\right)}_{v_{k}}
$$

where $v_{k}^{v}$ and $v_{k}^{o}$ are control action that leaves $\Omega^{v}$ respectively $\Omega^{o}$ invariant (those actions exist due to the controlled invariance properties). Thus $u_{k}$ is applied to the system (1) to compute $x_{k+1}$. Then, the IBT will be implemented with the 3 stages procedure.

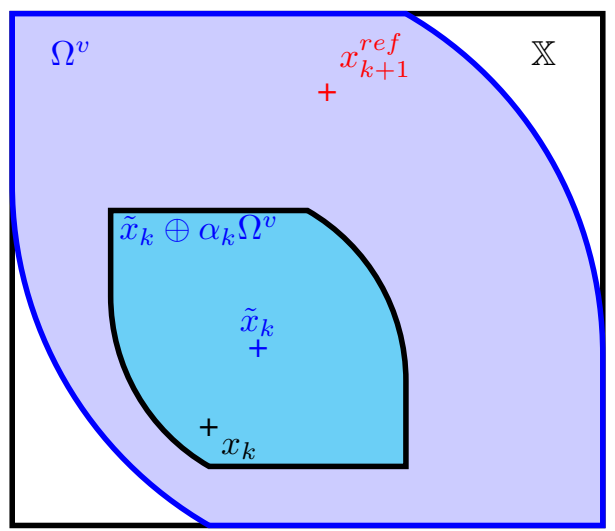

Fig. 4. IBC for Tracking Geometric interpretation

\section{Feasibility and stability properties}

First, let remind a characterization of convex sets based on Minkowski sums that will be used further :

A set $C$ is convex iff $\forall \lambda \in[0,1] \lambda C \oplus(1-\lambda) C=C$

Lemma III.2 (Convex complementary with Minkowski sum). Let $C$ a convex polyhedron , $x \in \mathbb{R}^{n}$ and $\alpha \in[0,1]$.

$$
\{\tilde{x}\} \oplus \alpha C \subset C \Longleftrightarrow x \in(1-\alpha) C
$$

Proof:

$$
\{x /\{x\} \oplus \alpha C \subset C\}=C \ominus \alpha C
$$

and according to the previous characterization :

$$
\begin{aligned}
C \ominus \alpha C & =[(1-\alpha) C \oplus \alpha C] \ominus \alpha C \\
& =(1-\alpha) C \oplus \alpha C \ominus \alpha C \\
& =(1-\alpha) C
\end{aligned}
$$

Lemma III.3. For all $x_{0} \in \Omega^{v}$ there exists $\tilde{x}_{0} \in \Omega^{v}$ such that (7) is feasible.

Proof: We observe that there exists at least two feasible choices $\tilde{x}_{0}=0$ or $\tilde{x}_{0}=x_{0}$. If $\tilde{x}_{0}=0$, then $\alpha=1$ and any $\tilde{u}_{0} \in \mathbb{U}$ is a feasible choice. If $\tilde{x}_{0}=x_{0}$, then any $\alpha \in[0,1]$ and $\tilde{u}_{0}=0$ is a feasible choice.

Proposition III.1. Given $x_{k}, \tilde{x}_{k}$ and $x_{k}^{r e f}$, if (7) is feasible and $x_{k+1} \in\left\{\tilde{x}_{k+1 \mid k}\right\} \oplus \alpha_{k} \Omega^{v}$, then (7) is feasible for $x_{k+1}, \tilde{x}_{k+1}$ and any $x_{k+1}^{r e f}$.

Proof: Assume (7) is feasible at step $\mathrm{k}$ and $x_{k+1} \in$ $\left\{\tilde{x}_{k+1 \mid k}\right\} \oplus \alpha_{k} \Omega^{v}$. By considering the feasible choice : $\tilde{x}_{k+1 \mid k+1}=\tilde{x}_{k+1 \mid k}$, the feasible choice $\alpha_{k+1}=\alpha_{k} \in[0,1]$ can be considered.

$$
\left\{\tilde{x}_{k+1 \mid k}\right\} \oplus \alpha_{k} \Omega^{v} \subset \Omega^{v} \Rightarrow\left\{\tilde{x}_{k+1 \mid k+1}\right\} \oplus \alpha_{k+1} \Omega^{v}
$$

According to Lemma III.2 :

$$
\left\{\tilde{x}_{k+1 \mid k+1}\right\} \oplus \alpha_{k+1} \Omega^{v} \Rightarrow \tilde{x}_{k+1 \mid k+1} \in\left(1-\alpha_{k+1}\right) \Omega^{v}
$$

$\tilde{x}_{k+1 \mid k+1} \in\left(1-\alpha_{k+1}\right) \Omega^{v}$, so there exists $\tilde{u} \in\left(1-\alpha_{k+1}\right) \mathbb{U}$ such that :

$$
\tilde{x}_{k+2 \mid k+1}=A \tilde{x}_{k+1 \mid k+1}+B \tilde{u} \in\left(1-\alpha_{k+1}\right) \Omega^{v}
$$


Let $\tilde{u}_{k} \in\left(1-\alpha_{k+1}\right) \mathbb{U}$ such that $\tilde{x}_{k+2 \mid k+1}=A \tilde{x}_{k+1 \mid k+1}+$ $B \tilde{u} \in\left(1-\alpha_{k+1}\right) \Omega^{v}$. According to Lemma III.2 :

$\tilde{x}_{k+2 \mid k+1} \in\left(1-\alpha_{k+1}\right) \Omega^{v} \Rightarrow\left\{\tilde{x}_{k+2 \mid k+1}\right\} \oplus \alpha_{k+1} \Omega^{v} \subset \Omega^{v}$

and the proof is complete

Proposition III.2. If (7) is feasible, then (8) is feasible for the respective solutions $\alpha_{k}$ and $\tilde{u}_{k}$

Proof:

$$
\begin{aligned}
\varepsilon_{k+1} & =x_{k+1}-\tilde{x}_{k+1 \mid k+1} \\
& =A\left(x_{k+1}-\tilde{x}_{k \mid k+1}\right)+B\left(u_{k}-\tilde{u}_{k}\right) \\
& =A \varepsilon_{k}+B v_{k} \\
& =A\left(c_{k} \varepsilon_{k}^{v}+\left(1-c_{k}\right) \varepsilon_{k}^{o}\right)+B\left(c_{k} v_{k}^{v}+\left(1-c_{k}\right) v_{k}^{o}\right) \\
& =c_{k}\left(A \varepsilon_{k}^{v}+B v_{k}^{v}\right)+\left(1-c_{k}\right)\left(A \varepsilon_{k}^{o}+B v_{k}^{o}\right)
\end{aligned}
$$

$A \varepsilon_{k}^{v}+B v_{k}^{v} \in \alpha_{k} \Omega^{v}$ according to the invariance of $\alpha_{k} \Omega^{v}$ at step $\mathrm{k}$ and $A \varepsilon_{k}^{o}+B v_{k}^{o} \in \alpha_{k} \Omega^{o}$. We choose $c_{k+1}=$ $c_{k}, \varepsilon_{k+1}^{v}=A \varepsilon_{k}^{v}+B v_{k}^{v}, \varepsilon_{k+1}^{o}=A \varepsilon_{k}^{o}+B v_{k}^{o}$

Proposition III.3. If (7) and (8) are feasible, then $u_{k}$ computed in (9) satisfies $u_{k} \in \mathbb{U}$ and the condition $x_{k+1} \in$ $\left\{\tilde{x}_{k+1 \mid k}\right\} \oplus \alpha_{k} \Omega^{v}$ holds.

Proof: $u_{k}^{v}\left(\varepsilon_{k}^{v}\right) \in \alpha \mathbb{U}$ and $u_{k}^{o}\left(\varepsilon_{k}^{o}\right) \in \alpha \mathbb{U}$ according to Theorem III.1. Then their convex combination : $c_{k} u_{k}^{v}\left(\varepsilon_{k}^{v}\right)+$ $\left(1-c_{k}\right) u_{k}^{o}\left(\varepsilon_{k}^{o}\right) \in \alpha \mathbb{U}$. Thus, $u_{k}$ is a combination of two elements of convex complementary sets, so $u_{k} \in \mathbb{U}$.

$$
\begin{aligned}
x_{k+1}-\tilde{x}_{k+1}= & A x_{k}+B u_{k}-\left(A \tilde{x}_{k}+B \tilde{u}_{k}\right) \\
= & A\left(x_{k}-\tilde{x}_{k}\right)+ \\
& +B\left(\tilde{u}_{k}+c_{k} v_{k}^{v}+\left(1-c_{k}\right) v_{k}^{o}\right)-B \tilde{u}_{k} \\
= & A\left(x_{k}-\tilde{x}_{k}\right)+B\left(c_{k} v_{k}^{v}+\left(1-c_{k}\right) v_{k}^{o}\right)
\end{aligned}
$$

Due to constraints (7c) : $\left(x_{k}-\tilde{x}_{k}\right) \in \alpha_{k} \Omega^{v}$. Additionally the control action $c_{k} v_{k}^{v}+\left(1-c_{k}\right) v_{k}^{o}$ is constructed to leave the current error invariant in $\alpha_{k} \Omega^{v}$. Consequently $\left(x_{k+1}-\right.$ $\left.\tilde{x}_{k+1}\right) \in \alpha_{k} \Omega^{v}$.

Proposition III.4. The IBC for tracking procedure is recursively feasible.

Proof: Lemma III.3 guarantees the initialization of optimization (7). Let (7) be feasible at step $k$. Then (8) is feasible at step $k$ according to Proposition III. 2 and it implies that the condition : $x_{k+1} \in\left\{\tilde{x}_{k+1 \mid k}\right\} \oplus \alpha_{k} \Omega^{v}$ holds thanks to Proposition III.3. Consequently, (7) is feasible at step $k+1$ thanks to Proposition III.1.

\section{Discussion AND EXTENSION}

\section{A. Discussion}

The optimization (7) is a $\mathrm{QP}$ problem if $\Omega^{v}$ and $\Omega^{o}$ are polyhedrons and the complexity of the optimization arguments is $(m+1)$ and (8) is a bilinear programming problem of complexity $(2 m+1)$ can be rewritten in a LP problem as it is presented in [12] with the variable change $r_{k}^{v}=c_{k} \varepsilon_{k}^{v}:$

$$
\begin{aligned}
& {\left[\begin{array}{c}
r_{k}^{v} \\
c_{k}
\end{array}\right]\left(\varepsilon_{k}, \alpha_{k}\right)=\underset{\left(r_{k}^{v}, c_{k}\right)}{\arg \min } c_{k} } \\
& \text { subject to } \\
& F_{v} r_{k}^{v} \leq c_{k} g_{v}, \\
& F_{o}\left(\varepsilon_{k}-r_{k}^{v}\right) \leq\left(1-c_{k}\right) g_{o}, \\
& c_{k} \in[0,1]
\end{aligned}
$$

where $\Omega^{v}=\left\{x \in \mathbb{R}^{n} / F_{v} x \leq g_{v}\right\}$ and $\Omega^{o}=\{x \in$ $\left.\mathbb{R}^{n} / F_{o} x \leq g_{o}\right\}$ The choice of the initial virtual state $\tilde{x}_{0}$ is important for the behavior of the system, if $\tilde{x}_{0}=0$ then, the optimization problem (7) has more degrees of freedom to choose a higher scaling factor $\alpha_{k}$ and so make the regulation be preponderant at the beginning until the trajectory of the system (1) reaches the virtual reference where $\alpha_{k}$ is close to 0 . If $\tilde{x}_{0}=x_{0}$ then the optimization (7) is always preponderant if there is no perturbation. It can be noted that there is no weighting on the control action $\tilde{u}_{k}$ in (7) because it would be redundant with the contraint (7e). Assumptions on convexity and invariance of inner and outer sets could be hard to hold. Ideally inner and outer sets have to be constructed at each step and not just re-scaled as we presented but those constructions can be computationally heavy although less conservative. Then, constraints (6d) and (6f) depends directly on the complexity of the outer set. In the case this outer set is polyhedral, as linear constraints account to the number of vertices of the outer set, that would increase the complexity of the problem. We stress the fact that for real-time systems, it is not relevant to reconstruct sets at each step.

\section{B. Extension}

In the previous section, the virtual reference is computed at each step with the results of optimization problems of the previous step, thus the virtual trajectory is globally constrained to satisfy a the linear dynamics (1).

$$
\begin{aligned}
\underset{\left(\tilde{x_{k}}, \tilde{u}_{k}, \alpha_{k}\right)}{\operatorname{minimize}} & \left\|x_{k+1}^{r e f}-\tilde{x}_{k+1 \mid k}\right\|_{Q}^{2} \\
\text { subject to } & \tilde{x}_{k+1 \mid k}=A \tilde{x}_{k \mid k}+B \tilde{u}_{k}, \\
& x_{k} \in\left\{\tilde{x}_{k \mid k}\right\} \oplus \alpha_{k} \Omega^{v} \\
& \left\{\tilde{x}_{k \mid k}\right\} \oplus \alpha_{k} \Omega^{v} \subset \Omega^{v} \\
& \tilde{u}_{k} \in\left(1-\alpha_{k}\right) \mathbb{U} \\
& \left\{\tilde{x}_{k+1 \mid k}\right\} \oplus \alpha_{k} \Omega^{v} \subset \Omega^{v}
\end{aligned}
$$

Then the regulation is identical to the previous algorithm.

Theorem IV.1. The tracking procedure based on optimization (11) is recursively feasible.

Proof: The proof is the same as the previous procedure.

By finding the virtual state as an optimization variable in the problem (7), the procedure resets $\tilde{x}_{k}$ at each time step and thus the trajectory is less conservative and the controller more aggressive in the sense that arbitrary changes in the reference will be transmitted in the virtual reference. 


\section{Simulations ANd Analysis}

Consider the discrete-time linear system (1). Assume there exists a stabilizing linear feedback law :

$$
u_{k}=-K x_{k} \forall k \in \mathbb{N}
$$

where $K \in \mathbb{R}^{m \times n}$. We can chose for example, an LQR feedback law.

Definition V.1 (Maximal Positively Invariant and Admissible Set). The Maximal Positively Invariant Admissible Set $O_{\infty}(\mathbb{U}, \mathbb{X})$ is the largest positively invariant set that respect states and control constraints.

For a stabilizing linear feedback, there exists a finitely determined maximal polyhedral invariant set that can be constructed with a procedure given in [18].

Definition V.2 (N-Step Controllable Invariant Set). We define the $N$-Step Controllable set $C_{N}(P)$ with respect to the set $P$ as the set of all states for which it exists a control sequence such that the system reaches the set $P$ in at most $N$ steps $(N \in$ $\mathbb{N})$ along an admissible trajectory. For all $x_{0} \in C_{N}(P)$ there exists $\left(u_{0}, \ldots, u_{N-1}\right) \in \mathbb{U}^{N}$ such that, for all $i \in \llbracket 0, N-1 \rrbracket$ $x_{i+1}=A x_{i}+B u_{i} \in C_{N}(P)$ and $x_{N} \in P$.

First, we can construct the maximal positively and invariant set $O_{\infty}$ for the system (1) and the feedback (10) with the procedure given in [19] in a polyhedral form :

$$
O_{\infty}=\left\{x \in \mathbb{R}^{n} / F_{o} x \leq g_{o}\right\}
$$

Then a N-Step controllable invariant set $C_{N}\left(O_{\infty}\right)$ can be constructed according to the procedure given in [14] in a polyhedral form :

$$
C_{N}\left(O_{\infty}\right)=\left\{x \in \mathbb{R}^{n} / F_{N} x \leq g_{N}\right\}
$$

In the following we will apply the methodological developments with the particular choices: $\Omega^{o} \leftarrow O_{\infty}$ and $\Omega^{v} \leftarrow$ $C_{N}\left(O_{\infty}\right)$

\section{A. Details}

The model used in simulation is a double integrator model

$$
x_{k+1}=\left[\begin{array}{cc}
1 & 0.08 \\
0 & 1
\end{array}\right] x+\left[\begin{array}{c}
0.0032 \\
0.08
\end{array}\right] u
$$

subject to :

$$
\begin{aligned}
& -2.6 \leq x_{1} \leq 2.6 \\
& -3 \leq x_{2} \leq 3 \\
& -5 \leq u \leq 5
\end{aligned}
$$

The reference is a model-based trajectory that leaves the state constraints set. The initial state of the virtual admissible reference set to the origin. The initial state of the system is on the frontier of the controlled invariant $C_{N}\left(O_{\infty}\right)$ at a vertex state with no speed.

The Tracking IBC is implemented according to the procedure provided in the section III but applied to polyhedral sets $O_{\infty}$ as the inner set and $C_{N}\left(O_{\infty}\right)$ as the outer one.

At each step, the control contribution of the inner set $\alpha_{k} O_{\infty}$ is a linear feedback on the error $\varepsilon_{k}^{o}: u_{k}^{o}=-K \varepsilon_{k}^{o}$ and the contribution of the outer set is a vertex control law which is a solution of the optimization :

$$
\begin{aligned}
& {\left[\begin{array}{c}
u_{k}^{v} \\
\lambda_{k}
\end{array}\right]\left(\varepsilon_{k}^{v}, \alpha_{k}\right)=\underset{\left(v_{k}^{v}, \lambda_{k}\right)}{\operatorname{minimize}} \lambda_{k}} \\
& \text { subject to } \\
& A \varepsilon_{k}^{v}+B v_{k}^{v} \in \lambda_{k} \alpha_{k} C_{N} \text {, } \\
& v_{k}^{v} \in \alpha_{k} \mathbb{U} \\
& \lambda_{k} \in[0,1]
\end{aligned}
$$

\section{B. Performances}

Figures 5,6,7 present the simulations obtained for the numerical model. Figure 6 presents the controlled invariant $C_{N}\left(O_{\infty}\right)$ and the relative positions of the reference signal, virtual reference and the state trajectory. Figure 7 details the time dependence for the IBT and the extended IBT strategies allowing to observe the controller first finds a scaling factor $\alpha_{0}=1$ so the control action is equivalent to an IBC regulation to the origin. Higher the scaling factor, more conservative the reference governor. When the regulation manages to lead the system close to the virtual reference, the scaling factor decreases and the reference governor applies the whole control input and coincides with the system state. Reference governor control component is scaled by $\left(1-\alpha_{k}\right) \mathbb{U}$ and the IBC component is scaled by $\alpha_{k} \mathbb{U}$.

For the standard IBT, the virtual trajectory tends to move away from the frontier due to the conservativeness induced by, in one hand, the re-scaling of the outer set instead of its reconstruction and, on the other hand the imposed dynamics to the whole trajectory. The dynamics of the extended IBT solution is less constrained due to the reset of the current virtual state $\tilde{x}$ at each step. The tracking of the virtual reference is performant as expected for IBC the only level of conservativeness being introduced by the set constraints (11c-11d). However, the structural properties of the optimization-based tracking scheme is exclusively based on convex (LP/QP) optimization and thus the computational performances prove to be very attractive for embedded applications.

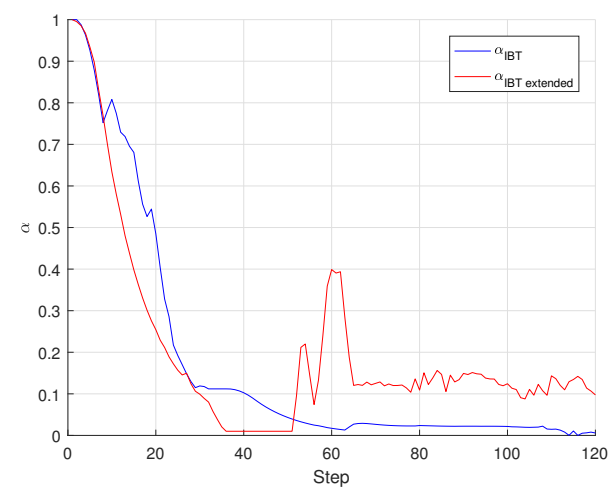

Fig. 5. Scaling factor $\alpha$ for IBT (blue) and for extended IBT (red) 

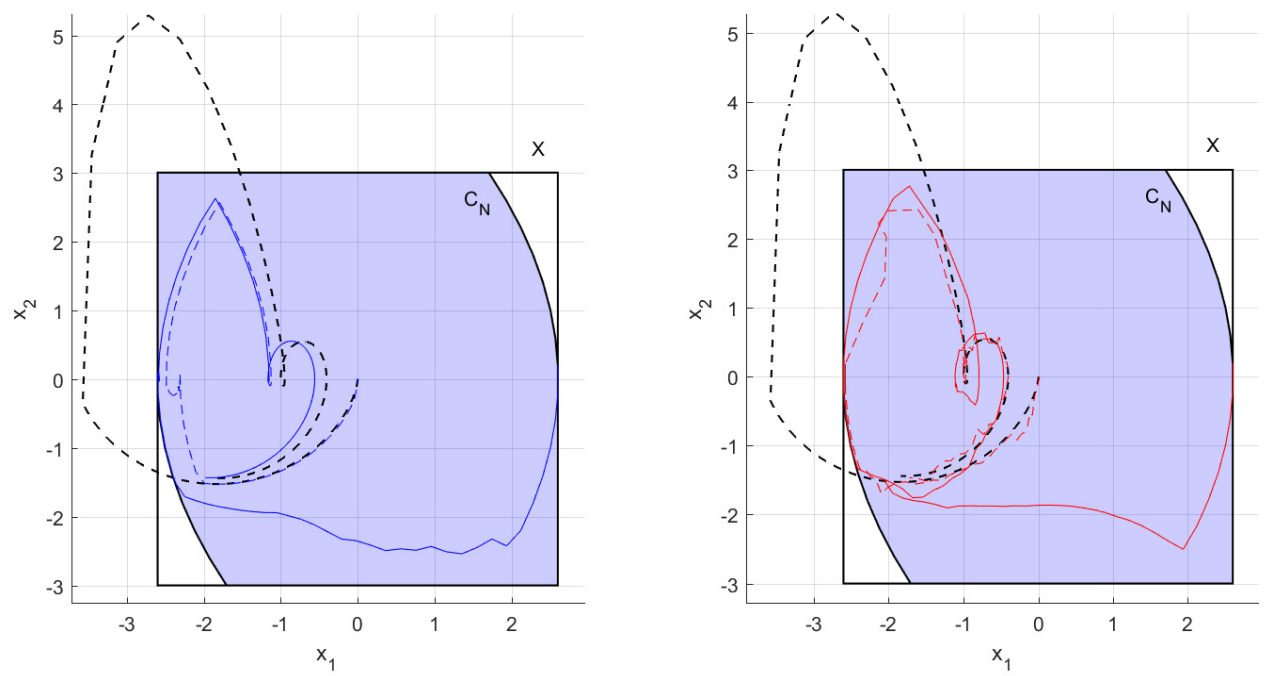

Fig. 6. Left : Trajectories for the IBT of the real reference $x^{r e f}$ (black, dashed),the virtual reference $\tilde{x}$ (blue, dashed) and the system $x$ (blue), Right : Trajectories for the extended IBT of the real reference $x^{r e f}$ (black, dashed),the virtual reference $\tilde{x}$ (red, dashed) and the system $x$ (red)
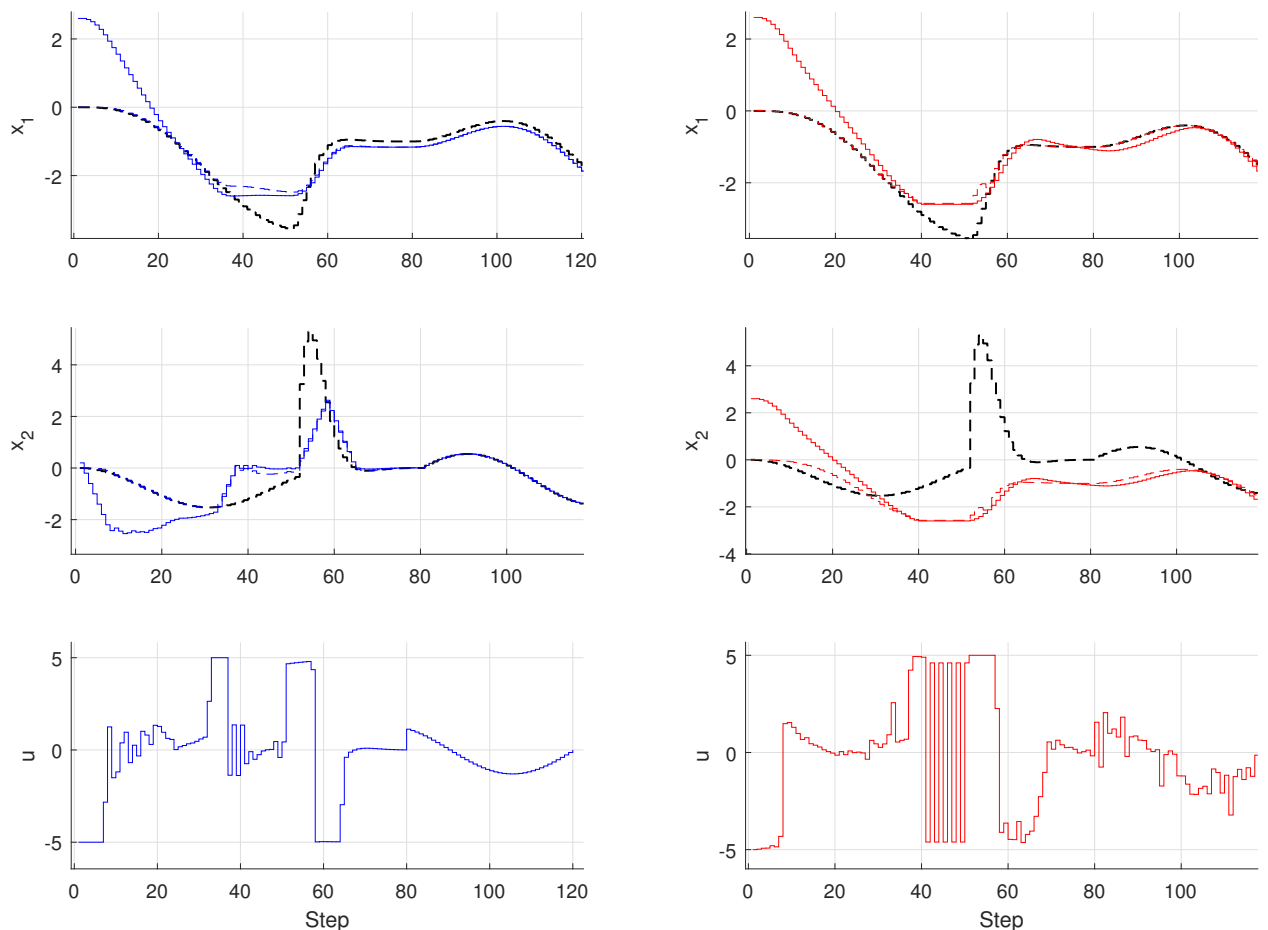

Fig. 7. Left column : state and input trajectories for IBT, Right column : state and input trajectories for extended IBT 


\section{CONCLUSION}

In this paper, a novel trajectory tracking procedure is presented for discrete-time linear systems with state and input constraints, designed from ingredients of generic IBC regulation (where controlled invariant outer and inner sets are considered) with an additional reference governor that guarantees the recursive feasibility of the entire procedure. A numerical example is also presented using high gain maximal output admissible set contained in a low gain controlled invariant set proving that all the ingredients of the tracking method can be readily constructed in terms of polyhedra, while the overall optimization-based implementation resides in a sequence of three convex optimization problems, confirming the compact and computational attractiveness of the interpolation-based control schemes for constrained control.

\section{REFERENCES}

[1] A. Bemporad, "Reference governor for constrained nonlinear systems," IEEE Transactions on Automatic Control, vol. 43, no. 3, pp. 415-419, 1998.

[2] D. Angeli and E. Mosca, "Command governors for constrained nonlinear systems," IEEE Transactions on Automatic Control, vol. 44, no. 4, pp. 816-820, 1999.

[3] E. Garone, S. Di Cairano, and I. Kolmanovsky, "Reference and command governors for systems with constraints: A survey on theory and applications," Automatica, vol. 75, pp. 306-328, 2017.

[4] D. Limon and T. Alamo, "Tracking model predictive control," Encyclopedia of Systems and Control, pp. 1-12, 2013.

[5] P. Falugi, "Model predictive control for tracking randomly varying references," International Journal of Control, vol. 88, no. 4, pp. 745753, 2015.

[6] L. Chisci and G. Zappa, "Dual mode predictive tracking of piecewise constant references for constrained linear systems," International Journal of Control, vol. 76, no. 1, pp. 61-72, 2003.

[7] S. Olaru and D. Dumur, "Compact explicit mpc with guarantee of feasibility for tracking," in Proceedings of the 44th IEEE Conference on Decision and Control. IEEE, 2005, pp. 969-974.

[8] D. Limon, I. Alvarado, T. Alamo, and E. Camacho, "Mpc for tracking of piece-wise constant references for constrained linear systems," IFAC Proceedings Volumes, vol. 38, no. 1, pp. 135-140, 2005.

[9] H.-N. Nguyen, "Constrained control of uncertain, time-varying, discrete-time systems," Lecture Notes in Control and Infomration Sciences, vol. 451, p. 17, 2014.

[10] H. N. Nguyen, "Commande sous contraintes pour des systèmes dynamiques incertains: une approache basée sur l'interpolation,” Ph.D. dissertation, 2012.

[11] P. . Gutman and M. Cwikel, "Admissible sets and feedback control for discrete-time linear dynamical systems with bounded controls and states," IEEE Transactions on Automatic Control, vol. 31, no. 4, pp. 373-376, April 1986.

[12] H.-N. Nguyen, P.-O. Gutman, S. Olaru, and M. Hovd, "Implicit improved vertex control for uncertain, time-varying linear discretetime systems with state and control constraints," Automatica, vol. 49, no. 9, pp. 2754-2759, 2013.

[13] F. Blanchini, "Ultimate boundedness control for uncertain discretetime systems via set-induced lyapunov functions," IEEE Transactions on automatic control, vol. 39, no. 2, pp. 428-433, 1994.

[14] H. Nguyen, P.-O. Gutman, S. Olaru, M. Hovd, and F. Colledani, "Improved vertex control for time-varying and uncertain linear discretetime systems with control and state constraints," in Proceedings of the 2011 American Control Conference. IEEE, 2011, pp. 4386-4391.

[15] I. Ballesteros-Tolosana, S. Olaru, P. Rodriguez-Ayerbe, G. Pita-Gil, and R. Deborne, "Comparison of optimization-based strategies for constrained control of auto-steering systems," in 2016 European Control Conference (ECC). IEEE, 2016, pp. 1592-1597.

[16] A. Tuchner and J. Haddad, "Vehicle platoon formation using interpolating control: A laboratory experimental analysis," Transportation Research Part C: Emerging Technologies, vol. 84, pp. 21-47, 2017.
[17] S. Scialanga and K. Ampountolas, "Interpolating control toolbox (ict)," in 2019 18th European Control Conference (ECC). IEEE, 2019, pp. 2510-2515.

[18] F. Blanchini and S. Miani, Set-theoretic methods in control. Springer, 2008.

[19] E. G. Gilbert and K. T. Tan, "Linear systems with state and control constraints: The theory and application of maximal output admissible sets," IEEE Transactions on Automatic control, vol. 36, no. 9, pp. 1008-1020, 1991. 